Winter Meeting, 8-9 December 2015, Roles of sleep and circadian rhythms in the origin and nutritional management of obesity and metabolic disease

\title{
Use of a Web-Based Dietary Assessment Tool in Early Pregnancy
}

\author{
L. Mullaney ${ }^{1}$, A. O’Higgins ${ }^{2}$, S. Cawley ${ }^{1}$, R. Kennedy ${ }^{1}$, D. McCartney ${ }^{1}$ and M.J. Turner ${ }^{2}$ \\ ${ }^{1}$ School of Biological Sciences, Dublin Institute of Technology, Dublin 8, Republic of Ireland and ${ }^{2} U C D$ Centre for \\ Human Reproduction, Coombe Women and Infants University Hospital, Dublin 8, Republic of Ireland
}

Given the importance of maternal diet in fetal development and in lifelong health outcomes ${ }^{1}$, accurate dietary assessment and interpretation is important for the derivation of efficacious, evidence-based nutritional interventions. In this context, dietary quality indices in pregnancy should be explicitly underpinned by data correlating food intake patterns with nutrient intakes known to be important for gestation.

Our aim was to assess the correlation between scores derived from a novel online Dietary Assessment Tool (DAT) and nutrient intake data derived from the previously validated Willett Food Frequency questionnaire (WFFQ). Women completed the validated WFFQ and online DAT questionnaire in their first trimester.

Positive correlations were observed between respondents' $(n=402)$ diet and nutrition scores derived from the online DAT, and their folate, vitamin $\mathrm{B}_{12}$, iron, calcium, and zinc intakes/MJ of energy consumed derived from the WFFQ (all $P<0 \cdot 001$ ). Negative correlations were observed between participants' diet and nutrition scores and their total energy intake $(P=0.04)$, and their percentage energy from fat, saturated fat, and non-milk extrinsic sugars (NMES) (all $P \leqslant 0 \cdot 001$ ). Median dietary fibre, beta carotene, folate, vitamin $\mathrm{C}$ and vitamin $\mathrm{D}$ intakes derived from the WFFQ, generally increased across quartiles of diet and nutrition score (all $P<0.001$ ).

Scores generated by this web-based dietary assessment tool correlate with important nutrient intakes in pregnancy, supporting its use in estimating overall dietary quality among obstetric populations. The technological advantages and potential interactive aspect of the DAT make it useful for collecting dietary information and could be linked to individualised advice on dietary intakes and lifestyle behaviours.

1. Langley-Evans SC (2014) Nutrition in early life and the programming of adult disease: a review. J Hum Nutr Diet 28;1-14. 